Konrad-Zuse-Zentrum

für Informationstechnik Berlin

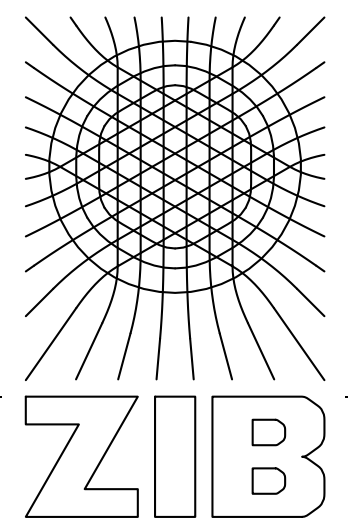

Takustraße 7

D-14195 Berlin-Dahlem

Germany

\author{
RALF BORNDÖRFER CHRISTIAN LIEBCHEN
}

\title{
When Periodic Timetables are Suboptimal
}




\title{
When Periodic Timetables are Suboptimal*
}

\author{
Ralf Borndörfer and Christian Liebchen
}

Konrad-Zuse-Zentrum für Informationstechnik Berlin, Takustr. 7, 14195

Berlin-Dahlem, Germany

Technische Universität Berlin, Institut für Mathematik, Str. des 17. Juni 136, 10623 Berlin, Germany

Email borndoerfer@zib.de, liebchen@math.tu-berlin.de

Summary. "The timetable is the essence of the service offered by any provider of public transport" (Jonathan Tyler, CASPT 2006). Indeed, the timetable has a major impact on both operating costs and on passenger comfort. Most European agglomerations and railways use periodic timetables in which operation repeats in regular intervals. In contrast, many North and South American municipalities use trip timetables in which the vehicle trips are scheduled individually subject to frequency constraints. We compare these two strategies with respect to vehicle operation costs. It turns out that for short time horizons, periodic timetabling can be suboptimal; for sufficiently long time horizons, however, periodic timetabling can always be done 'in an optimal way'.

\section{Introduction}

The construction of the timetable is perhaps the most important scheduling activity of a railway or public transport company. It has a major impact on operating costs and on passenger comfort. The problem has been extensively covered in the operations research literature, see [2] for a recent survey. There are two main timetabling strategies that differ w.r.t. to structural dependencies between individual trips. In a periodic timetable, there is a fixed time interval between two trips; if a single trip is scheduled on a directed line, all other trips of this line are determined. In contrast, in a trip timetable, each trip is scheduled individually, subject to frequency constraints. Stipulating appropriate constraints, a trip timetable can be forced to become periodic, or, to put it the other way round, trip timetables feature more degrees of freedom than periodic ones. We investigate in this paper the question whether this freedom can be used to lower operation costs in terms of numbers of vehicles. Of course, such improvements (if any) come at the price of diminishing the regularity of the timetable.

\footnotetext{
* Supported by the DFG Research Center Matheon (http://www.matheon.de).
} 


\section{The Timetabling Problem}

We consider the timetabling problem for a single, bidirectional line between two stations $A$ and $B$. The line is operated by homogenous vehicles with running times $t_{a b}$ and $t_{b a}$ in directions $A \rightarrow B$ and $B \rightarrow A$, respectively (these include minimum turnaround times in the respective terminus stations). We want to construct a timetable that covers $N$ time periods of length $T$ with a trip frequency of $f$ vehicles per time period, and such that the minimum headway between two consecutive trips is at least $\ell$ and at most $u$. We assume that $f$ divides $T$ and call $T / f$ the period time of the timetable (to be constructed). We further assume $\ell \leq T / f \leq u \leq T$ and that all mentioned numbers are positive integers except for $\ell$, which is supposed to be a non-negative integer. The timetable that we want to construct involves $m:=N \cdot f$ departures at station $A$, that we denote by $U=\left\{u_{1}, \ldots, u_{m}\right\}$, and the same number of departures at station $B$, that we denote by $V=\left\{v_{1}, \ldots, v_{m}\right\}$; let $U \cup V$ be the set of all these departure events. A timetable is a function $t: U \cup V \mapsto \mathbb{Z}$ that maps departures to times such that the following conditions hold:

$$
\begin{array}{lll}
\text { (i) } \quad & t\left(u_{i}\right) \leq t\left(u_{i+1}\right) & i=1, \ldots, m-1 \\
& t\left(v_{i}\right) \leq t\left(v_{i+1}\right) & i=1, \ldots, m-1 \\
\text { (ii) } \quad & \lfloor(i-1) / f\rfloor T \leq t\left(u_{i}\right)<(\lfloor(i-1) / f\rfloor+1) T & i=1, \ldots, m-1 \\
& \lfloor(i-1) / f\rfloor T \leq t\left(v_{i}\right)<(\lfloor(i-1) / f\rfloor+1) T & i=1, \ldots, m-1 \\
\text { (iii) } \quad \ell \leq t\left(u_{i+1}\right)-t\left(u_{i}\right) \leq u, & i=1, \ldots, m-1 \\
& \ell \leq t\left(v_{i+1}\right)-t\left(v_{i}\right) \leq u, & i=1, \ldots, m-1 .
\end{array}
$$

Constraints (i) ensure that the departure times at both stations ascend in time, (ii) guarantees $f$ departures in each period at each station, and (iii) enforce a minimum and maximum headway of $\ell$ and $u$ between two consecutive departures of trips. A timetable is a periodic timetable if condition (iii) is replaced by

$$
t\left(u_{i+1}\right)-t\left(u_{i}\right)=t\left(v_{i+1}\right)-t\left(v_{i}\right)=T / f, \quad i=1, \ldots, m-1,
$$

otherwise it is a trip timetable. Note that a timetable can be forced to be periodic by stipulating $\ell=T / f=u$. The timetabling problem is to determine a feasible timetable that can be operated with a minium number of vehicles?

We remark that our problem definition deliberately omits technical constraints, such as passing sidings, in order to focus upon purely structural implications.

\footnotetext{
${ }^{2}$ This definition is made in our context; there are other types of timetabling problems in the literature.
} 


\section{Periodic vs. Trip Timetables}

Lemma 1. Consider a public transport line between stations $A$ and $B$ with running times $t_{a b}$ and $t_{b a}$ which include the minimum turnaround times in the respective terminus stations, such that $t_{a b}+t_{b a}$ is an integer multiple of $T$. Then, operating this line for a time duration of at least $N \cdot T>t_{a b}+t_{b a}$ requires at least

$$
Z:=\frac{t_{a b}+t_{b a}}{\frac{T}{f}}
$$

vehicles in an arbitrary timetable.

Proof. At least $f$ vehicles have to be scheduled in each of the first $Z / f$ time periods until the first vehicle can be reused for a second trip in the same direction.

Lemma 2. Consider a public transport line between stations $A$ and $B$ with running times $t_{a b}$ and $t_{b a}$ (again including minimum turnaround times). $O p$ erating this line at shorter running times $t_{a b}^{\prime} \leq t_{a b}$ and $t_{b a}^{\prime} \leq t_{b a}$ does not increase the number of vehicles that are required for operation in the respectively best arbitrary timetables for this line.

Proof. For the optimal timetables for running times $t_{a b}$ and $t_{b a}$ there exist timetables with running times $t_{a b}^{\prime}$ and $t_{b a}^{\prime}$ that can be operated at the same number of vehicles. In fact, add a turnaround waiting time at terminus station $B$ of $t_{a b}-t_{a b}^{\prime}$, and a waiting time of $t_{b a}-t_{b a}^{\prime}$ at station $A$.

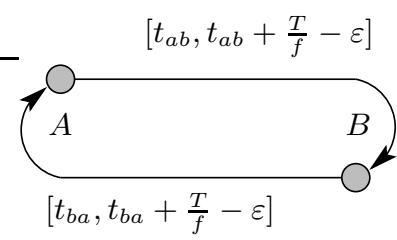

Fig. 1. The so-called PESP-graph (see, e.g., 2]) for the situation of the periodic vehicle circulation as it is considered in Prop. 1]

Proposition 1. Consider a public transport line between stations $A$ and $B$ with a period time $\frac{T}{f}$ and running times $t_{a b}$ and $t_{b a}$ (again including minimum turnaround times in the terminus stations) such that $t_{a b}+t_{b a}<N T-T / f$. Then, any periodic timetable requires at least

$$
Z_{0}:=\left\lceil\frac{t_{a b}+t_{b a}}{\frac{T}{f}}\right\rceil
$$

vehicles for operation. Moreover, any periodic timetable for this line can be operated with at most $Z_{0}+1$ vehicles. Indeed, there exist periodic timetables that can be operated with $Z_{0}$ vehicles. 
Proof. Using the cycle inequalities due to Odijk 4, it had been observed by Nachtigall [3] that there exists some appropriate $\varepsilon>0$ such that the following general bounds on the number $Z$ of vehicles are valid for all periodic timetables, and tight for some timetables:

$$
Z_{0}:=\left\lceil\frac{t_{a b}+t_{b a}}{\frac{T}{f}}\right\rceil \leq Z \leq\left\lfloor\frac{t_{a b}+\frac{T}{f}-\varepsilon+t_{b a}+\frac{T}{f}-\varepsilon}{\frac{T}{f}}\right\rfloor \leq Z_{0}+1 .
$$

Lemma 3. Consider a public transport line between stations $A$ and $B$ with running times $t_{a b}$ and $t_{b a}$ which once more include the minimum turnaround times in the respective terminus stations. Then, operating this line for at least a time duration of $N \cdot T>t_{a b}+t_{b a}$ requires at least

$$
Z_{1}:=\left\lfloor\frac{t_{a b}+t_{b a}}{T}\right\rfloor \cdot f
$$

vehicles in an arbitrary timetable.

Theorem 1. For each public transportation line with running times $t_{a b}$ and $t_{b a}$ (including minimum turnaround times), there exists a number $N_{0} \in \mathbb{N}$ such that operating the line for a time duration of at least $N_{0} \cdot T$ requires at least

$$
Z_{0}=\left\lceil\frac{t_{a b}+t_{b a}}{\frac{T}{f}}\right\rceil
$$

vehicles for operation. In other words, for sufficiently long time horizons, the minimum number of vehicles needed to operate a trip timetable is equal to the minimum number of vehicles needed to operate a periodic timetable.

Proof. In a time duration of $N \cdot T$, for the two directions of the line together there must be scheduled at least $2 \mathrm{Nf}$ trips. In turn, one vehicle can cover no more than $\left\lceil\frac{2 N T}{t_{a b}+t_{b a}}\right\rceil$ of these trips.

Now, choose $N_{0}$ such that $\frac{2 N_{0} T}{t_{a b}+t_{b a}}$ becomes integer. Then, the number of required vehicles is bounded from below by

$$
\begin{aligned}
\frac{2 N_{0} f}{\frac{2 N_{0} T}{t_{a b}+t_{b a}}} & =\frac{f}{T} \cdot\left(t_{a b}+t_{b a}\right) \\
& =\frac{t_{a b}+t_{b a}}{\frac{T}{f}} .
\end{aligned}
$$

Since we must only consider integer quantities of vehicles, (5) follows. 


\section{Example}

Let the balancing intervalequal $T=60$ minutes, and let the number of required trips within this interval be $f=3$. We consider a parameterized oneway running time $t_{a b}=t_{b a}=60-c$, which includes the minimum turnaround times, for $c \in\{1,2, \ldots, 10\}$.

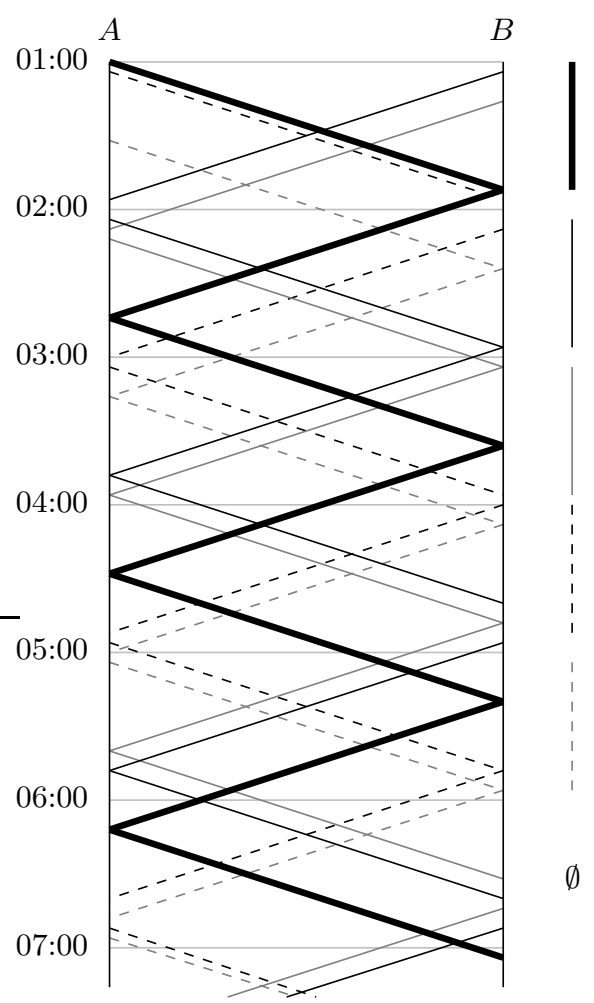

Fig. 2. A timetable for $t_{a b}=t_{b a}=52$, that uses only five vehicles. In the first five hours, there are three departures from each terminus station. But in the sixth hour, there are only two departures from $B$, which makes this timetable infeasible for $N=6$. The lines on the right indicate the vehicle that covers two trips in the corresponding hour.

First, observe that whenever $c<10$, then the number of vehicles that are required in the best periodic timetables equals

$$
Z_{0}=\left\lceil\frac{t_{a b}+t_{b a}}{\frac{T}{f}}\right\rceil=\left\lceil\frac{120-2 c}{\frac{60}{3}}\right\rceil=\left\lceil\frac{120-2 c}{20}\right\rceil=\left\lceil\frac{60-c}{10}\right\rceil=6 .
$$

Second, it can be verified that in any trip timetable, we need at least five vehicles for operating this line over at least three hours. Last, recall from 
the proof of Theorem 1 that after at least $100-2 c$ hours we can be sure to also require six vehicles when scheduling each trip individually. Yet, in this example we will show that, for certain running times, as early as after at least six hours we are sure to need the sixth vehicle also in any trip timetable.

The first simple observation is that within each hour, when considering both directions of the line together, there must be six trips in the schedule. Hence, in order to need only five vehicles, there must be one vehicle within each hour that covers two of these six trips. Of course, these two trips must be in opposite direction.

Now comes the key observation: If some fixed vehicle covers two trips in hour $X$, then it cannot cover two trips in any of the hours $\{X+1, \ldots, X+$ $\left.\left\lfloor\frac{T}{c}\right\rfloor-2\right\}$. As a consequence, if the one-way running time was $t_{a b}=52$, and thus $c=8$, then no vehicle can cover two trips in two of the hours $\{1, \ldots, 6\}$, because of $X+\left\lfloor\frac{T}{c}\right\rfloor-2=1+7-2=6$. Hence, in the sixth hour of operation, the latest, a sixth vehicle has to be put into operation, cf. Fig. 2.

\section{Passenger Waiting Times}

One can also analyze the differences between periodic and trip timetables with respect to passenger comfort, i.e., waiting times. An analysis of this type can be found in [1. There, an example of a transportation network is given, in which passengers spend strictly less waiting time at transfers, if a trip timetable is used instead of when a periodic timetable is used.

\section{References}

1. Christian Liebchen. Fahrplanoptimierung im Personenverkehr-Muss es immer ITF sein? Eisenbahntechnische Rundschau, 54(11):689-702, 2005. In German.

2. Christian Liebchen. Periodic Timetable Optimization in Public Transport. Ph.D.thesis, Technische Universität Berlin, 2006. dissertation.de-Verlag im Internet.

3. Karl Nachtigall. Periodic Network Optimization and Fixed Interval Timetables. Habilitation thesis, Universität Hildesheim, 1998.

4. Michiel A. Odijk. A constraint generation algorithm for the construction of periodic railway timetables. Transportation Research B, 30(6):455-464, 1996. 